
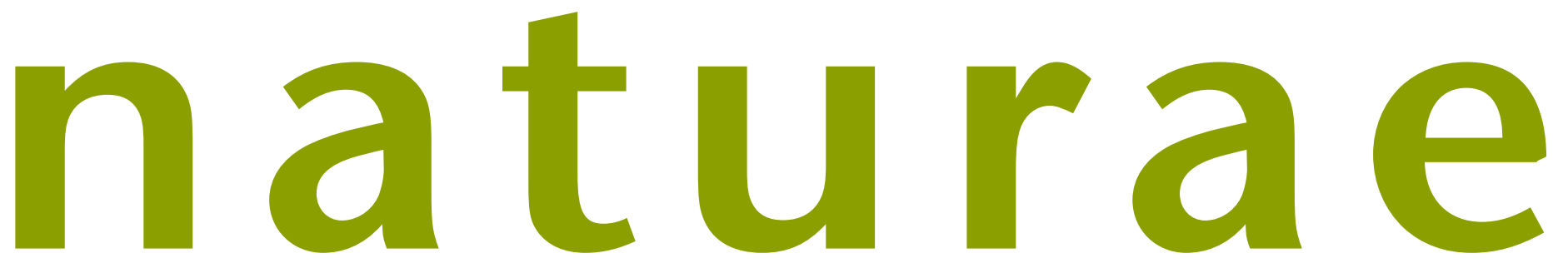

$2021 \cdot 3$

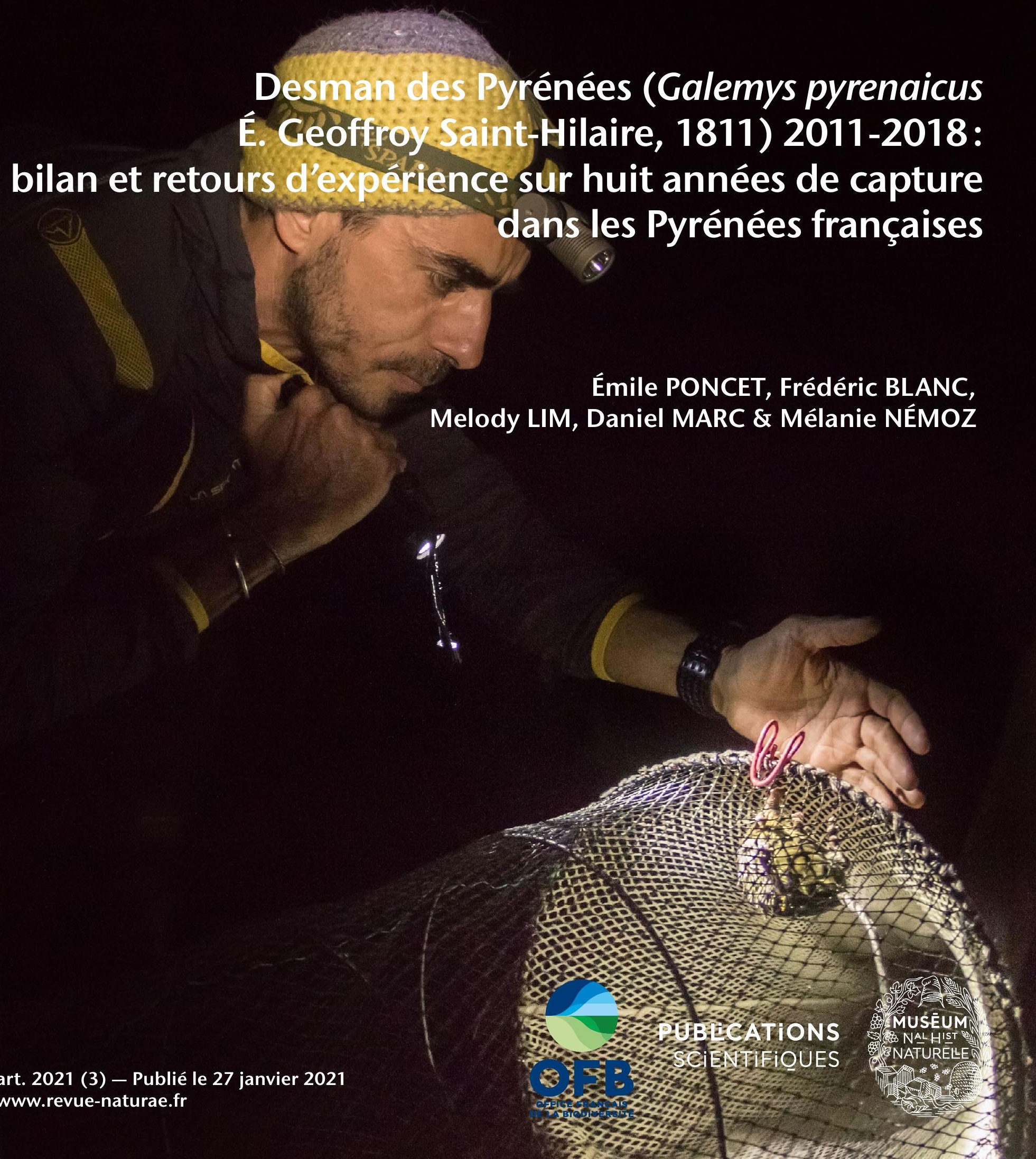


DiRECTEUR DE LA PUBLICATION/PUBLICATION DIRECTOR: Bruno David,

Président du Muséum national d'Histoire naturelle

RÉDACTEUR EN CHEF/EDITOR-IN-CHIEF: Jean-Philippe Siblet

ASSISTANTE DE RÉDACTION/ASSISTANT EDITOR: Sarah Figuet (naturae@mnhn.fr)

MISE EN PAGE/PAGE LAYOUT: Sarah Figuet

COMITÉ SCIENTIFIQUE/SCIENTIFIC BOARD:

Luc Abbadie (UPMC, Paris)

Luc Barbier (Parc naturel régional des caps et marais d'Opale, Colembert)

Aurélien Besnard (CEFE, Montpellier)

Vincent Boullet (Expert indépendant flore/végétation, Frugières-le-Pin)

Hervé Brustel (École d'ingénieurs de Purpan, Toulouse)

Patrick De Wever (MNHN, Paris)

Thierry Dutoit (UMR CNRS IMBE, Avignon)

Éric Feunteun (MNHN, Dinard)

Romain Garrouste (MNHN, Paris)

Grégoire Gautier (DRAAF Occitanie, Toulouse)

Olivier Gilg (Réserves naturelles de France, Dijon)

Frédéric Gosselin (Irstea, Nogent-sur-Vernisson)

Patrick Haffner (UMS PatriNat, Paris)

Frédéric Hendoux (MNHN, Paris)

Xavier Houard (OPIE, Guyancourt)

Isabelle Leviol (MNHN, Concarneau)

Francis Meunier (Conservatoire d'espaces naturels - Hauts-de-France, Amiens)

Serge Muller (MNHN, Paris)

Francis Olivereau (DREAL Centre, Orléans)

Laurent Poncet (UMS PatriNat, Paris)

Nicolas Poulet (OFB, Vincennes)

Jean-Philippe Siblet (UMS PatriNat, Paris)

Laurent Tillon (ONF, Paris)

Julien Touroult (UMS PatriNat, Paris)

Couverture/COVER:

Capture d'un Desman des Pyrénées, Galemys pyrenaicus É. Geoffroy Saint-Hilaire, 1811. Crédit photo: Lucas Santucci - agence Zeppelin.

Naturae est une revue en flux continu publiée par les Publications scientifiques du Muséum, Paris

Naturae is a fast track journal published by the Museum Science Press, Paris

Les Publications scientifiques du Muséum publient aussi / The Museum Science Press also publish:

Adansonia, Zoosystema, Anthropozoologica, European Journal of Taxonomy, Geodiversitas, Cryptogamie sous-sections Algologie, Bryologie, Mycologie, Comptes Rendus Palevol.

Diffusion - Publications scientifiques Muséum national d'Histoire naturelle

CP 41 - 57 rue Cuvier F-75231 Paris cedex 05 (France)

Tél.: 33 (0)1 407948 05/Fax: 33 (0)1 40793840

diff.pub@mnhn.fr/http://sciencepress.mnhn.f

(C) Publications scientifiques du Muséum national d'Histoire naturelle, Paris, 2021

ISSN (électronique/electronic): 1638-9387 


\title{
Desman des Pyrénées (Galemys pyrenaicus É. Geoffroy Saint-Hilaire, 1811) 2011-2018: bilan et retours d'expérience sur huit années de capture dans les Pyrénées françaises
}

\author{
Émile PONCET \\ Frédéric BLANC \\ Melody LIM \\ Daniel MARC \\ Mélanie NÉMOZ \\ Conservatoire d'Espaces naturels d'Occitanie, \\ 75 voie du TOEC, BP 57611, F-31076 Toulouse cedex 3 (France). \\ emile.poncet@cen-occitanie.org \\ frederic.blanc@cen-occitanie.org \\ melody.lim@cen-occitanie.org \\ daniel.marc@cen-occitanie.org \\ melanie.nemoz@cen-occitanie.org
}

Soumis le 19 mai 2020 | Accepté le 24 septembre 2020 | Publié le 27 janvier 2021

Poncet É., Blanc F., Lim M., Marc D. \& Némoz M. 2021. - Desman des Pyrénées (Galemys pyrenaicus É. Geoffroy Saint-Hilaire, 1811) 2011-2018: bilan et retours d'expérience sur huit années de capture dans les Pyrénées françaises. Naturae 2021 (3): 35-47. https://doi.org/10.5852/naturae2021a3

\section{RÉSUMÉ}

À l'heure où la prise en compte du Desman des Pyrénées, Galemys pyrenaicus É. Geoffroy Saint-Hilaire, 1811 dans toutes les procédures environnementales devient la norme, il convient de proposer des protocoles efficaces pour permettre le suivi de l'espèce. Outre la recherche de feces, d'autres techniques ont été testées en France ces dernières années, parmi elles les radeaux à empreintes, l'ADN environnemental, les tunnels à crottes et enfin la capture. Celle-ci peut constituer une alternative à la détection de l'espèce, notamment sur les secteurs ou la recherche de feces est inefficace à cause de variations hydrologiques, de la morphologie des sites ou du comportement de l'espèce, mais est aussi indispensable à certains protocoles tels que les suivis par radiopistage ou dans certains cas les CMR. En s'inspirant d'une méthode élaborée depuis la fin du XIXe siècle, 80 opérations de capture ont été menées dans les Pyrénées françaises depuis 2011 sur 24 cours d'eau ou lacs différents pour un résultat de 57 desmans capturés. Le bilan présenté ici propose un retour d'expérience sur des éléments du protocole et une analyse de la plus-value de la méthode par rapport à la recherche de feces classique. En l'état actuel, aucun lien significatif n’apparaît entre la réussite des opérations et les variables expérimentales que sont le nombre de pièges posés et la durée de l'opération (nuit complète ou nuit partielle). En revanche, le linéaire de piégeage semble avoir un effet avec un nombre de captures plus élevé pour des linéaires supérieurs à 500 mètres. Cela pourrait indiquer que l'efficacité d'une capture soit optimisée en couvrant un plus grand linéaire et donc davantage de domaines vitaux potentiels. Ensuite, si la durée de l'opération ne semble pas impacter directement les résultats, il apparaît primordial de ne pas interrompre une opération en cours de nuit, du moins tant que ses objectifs ne sont pas remplis. En effet, des captures peuvent intervenir à toute heure de la nuit avec notamment deux pics qui correspondent aux pics d'activité de plusieurs desmans suivis en radiopistage. Des captures diurnes sont aussi possibles. Par ailleurs, une analyse détaillée des taux de captures pour chaque type de nasses utilisées montre que les nasses à ailettes présentent la meilleure efficacité bien

MOTS-CLÉS

Desman des Pyrénées, détection, piégeage, protocole approche comparative. que les nasses souples simples aient un meilleur rapport efficacité/ coût. Ces deux modèles sont donc à privilégier. Enfin, toutes ces opérations ne permettent pas de mettre en évidence une plus-value globale de la méthode pour la détection de l'espèce par rapport à la recherche de feces. Cela pourrait toutefois être le cas sur des milieux lacustres avec davantage de résultats positifs à la capture mais le nombre de réplicas demeure encore trop faible pour valider cette hypothèse. 


\author{
KEY-WORDS \\ Pyrenean Desman, \\ detection, \\ trapping, \\ protocol, \\ comparative approach.
}

\begin{abstract}
Pyrenean Desman (Galemys pyrenaicus É. Geoffroy Saint-Hilaire, 1811) 2011-2018, assessment and feedback on eight years of trapping in the French Pyrenees.

At a time when the Pyrenean Desman's consideration becomes systematic in all environmental procedures, we have to purpose efficient protocols in order to allow the species monitoring. More than faeces research, several approaches were tested in France over the past years as tracks rafts, environmental DNA, faeces tunnels and finally trapping. This one can be an alternative way to detect the species, especially on some sectors where the faeces research is inefficient due to hydrological variations, sectors morphology or species behavior. It is also essential for some protocols as radiotracking or CMR. Based on the method developed since the end of the $19^{\text {th }}$ century, 80 trapping operations were carried in the French Pyrenees since 2011. They were distributed on 24 different rivers or lakes for a result of 57 trapped desmans. The assessment presented in this document purpose a feedback on the protocol elements and an added value analysis of this protocol in comparison with the faeces research. As things stand currently, there is no significant link between the success of the operations and the experimental variables which are the number of traps set and the duration of the operation (full night or partial night). However the trapping linear seems to have an effect on the success of the operations. Indeed, captures were more abundant when trapped linear is greater than 500 meters. This could indicate that the efficiency of a trapping operation would be better on a greater linear because it would cover several potential desmans home ranges. Moreover, even if the duration does not seem to have a direct impact on the results, it is important not to interrupt an operation during the night, at least as long as its objectives are not met. Indeed, captures can occur all night long and we notice two peaks of activities which are similar the ones of several desmans monitored by radiotracking. Daytime captures are also possible. In addition, a detailed analysis of the catch rates for each type of tested traps reveals that traps with winglets offer the better efficiency although simple and flexible traps are cheaper and easier to set and to carry. Therefore, these two types of traps have to be a first choice for trapping operations. Finally, all these operations do not enable to highlight a global added value of this protocol compared to faeces research for the species detection. This could however be the case on lake environments with more positive results. However, the number of replicas is still too low to validate this hypothesis.
\end{abstract}

\section{INTRODUCTION}

Espèce protégée et en régression sur l'ensemble de son aire de répartition, le Desman des Pyrénées (Galemys pyrenaicus É. Geoffroy Saint-Hilaire, 1811) fait l'objet d'une attention particulière avec plusieurs programmes de conservation. Le PNAD, Plan national d'Actions pour le Desman 20092014 (Némoz \& Bertrand 2008), a tout d'abord permis d'actualiser son aire de répartition française, de développer des outils d'étude non invasifs mais aussi de constituer et d'animer un réseau d'acteurs. Il est depuis 2014 relayé par le programme LIFE+ Desman (LIFE13NAT/FR/000092) qui vise à mettre en œuvre des mesures opérationnelles de gestion pour la sauvegarde de l'espèce. Enfin, à compter de 2021, les actions se poursuivront dans le cadre d'un second PNA, lequel est en cours de rédaction.

La mise en œuvre de ces actions conduit aujourd'hui à une meilleure prise en compte de l'espèce dans les différents projets d'aménagements en rivière susceptibles d'impacter ses populations ou son habitat. Cette évolution passe par la bonne prise en compte de l'espèce dans l'état initial et l'application de la procédure éviter, réduire, compenser, accompagner, suivre. À ces fins, les experts doivent fournir des outils et des protocoles standardisés et reconnus scientifiquement. La recherche de fèces est privilégiée et a fait l'objet d'une méthodologie validée et publiée (Charbonnel et al. 2017). Néanmoins, le Desman des Pyrénées est une espèce qui peut s'avérer difficilement détectable. Effectivement, si le protocole est construit de façon à limiter autant que possible la fausse absence, la recherche de fèces reste soumise à des aléas climatiques et hydrologiques, mais probablement aussi à la morphologie des cours d'eau ou à des variations comportementales pouvant la rendre difficile ou inefficace.

Ce constat a amené le Conservatoire d'Espaces naturels d'Occitanie et ses partenaires à tester des méthodes complémentaires pour optimiser la détection du Desman (radeaux à empreintes, $\mathrm{ADN}$ environnemental, tunnels à fèces, etc.). Les opérations de captures font également partie de ces méthodes. Outre l'objectif de valider la présence du Desman sur certains secteurs, la capture est aussi nécessaire pour l'acquisition de connaissances sur la biologie et l'écologie du Desman. Elle peut être utilisée dans des protocoles de capture-marquagerecapture (CMR) qui apportent des éléments sur la dynamique de population et elle est un préalable indispensable à tout suivi d'individus par radiopistage (domaine vital, localisation de gîtes, etc.).

En s'inspirant de travaux déjà réalisés, l'utilisation de pièges comportementaux de type «nasses» a été retenue. Nous proposons ici un bref historique de l'évolution de cette méthode de 1930 à nos jours. 
Dès le XIXe siècle et probablement avant même sa description scientifique, les desmans étaient piégés dans les nasses posées par les pêcheurs ou les braconniers (Trutat 1891) et ce sont eux qui ont fourni l'essentiel des individus étudiés par la science dans les décennies suivantes (Puisségur 1935). Les premières captures à des fins scientifiques interviennent en 1933 avec Puisségur sur le Lez en Ariège (Puisségur 1935). Il utilise des nasses métalliques à un ou deux compartiments avec un maillage carré de un centimètre. Il capture 17 individus pour un effort de capture de 36 nuits-pièges, soit un taux de capture de $47 \%$ avec parfois deux individus capturés simultanément dans un même piège. Malgré une tentative en hiver, tous les individus ont été capturés en été. Quatre cas de mortalité ont été constatés sans que la raison ne soit toutefois précisée. Puisségur installait ses pièges au crépuscule, dans des zones de courant, l'entrée orientée vers l'aval et avec une partie émergée. D'autres configurations ont été testées mais n'ont donné aucun résultat.

En 1956, Peyre reprend le même modèle de nasse à double compartiment que Puisségur mais adapte le système de piégeage (Peyre 1956). Une partie des pièges est toujours posée de façon isolée mais il expérimente aussi la pose en batterie. Dix pièges sont alors installés de front de manière à barrer intégralement toute la largeur du cours d'eau. Ils sont laissés dans le cours d'eau nuit et jour et ne sont relevés qu'au matin. En quatre ans, Peyre capture plus de 300 desmans répartis sur plusieurs rivières pyrénéennes. Il ne précise toutefois pas l'effort de capture déployé. Par ailleurs, en s'inspirant des travaux de Bouget en 1915 dans les Hautes-Pyrénées (Peyre 1956) qui n’a pas communiqué sur ces résultats, Puisségur et Peyre testent également le piégeage en berge avec des nasses appâtées ou non. Les résultats ne sont pas concluants car aucun desman n'est capturé. Des nasses appâtées sont aussi installées dans le cours d'eau mais ne permettent pas non plus de captures. Néanmoins, si Peyre ne précise pas l'appât utilisé, Bouget et Puisségur n'utilisent que des poissons (anguilles, chabots, barbillons, etc.). Or, il est désormais bien connu et admis que le Desman est une espèce strictement insectivore (Biffi et al. 2017) ce qui induit de relativiser les résultats du piégeage appâté tel qu'il était pratiqué par ces deux auteurs.

En 1973, Richard reprend également le même matériel mais apporte quelques améliorations aux nasses (Richard 1973). Sur les conseils de Peyre, il remplace d'abord le second compartiment par un long entonnoir également en métal qui repose sur la berge, puis par une boîte en bois au-dessus de la nasse pour permettre aux individus de se mettre au sec. Le poids des nasses devenant trop lourd, il est finalement le premier à remplacer la structure métallique des nasses par du nylon, bien plus léger, économique et potentiellement moins dangereux pour les desmans capturés. Les nouvelles nasses sont donc dotées d'un compartiment simple et d'un filet attaché à la berge. Il ne précise toutefois pas le taux de capture de ces nouvelles nasses. Enfin, le protocole de relève est modifié avec un contrôle des pièges au moins deux fois par nuit. La position des pièges vers l'aval et dans des veines de courant demeure la seule configuration efficace. La technique du piégeage est aussi utilisée en Espagne avec notamment Niethammer qui capture 27 desmans en quatre ans (Niethammer 1970). Il ne précise toutefois pas le matériel utilisé ni le protocole suivi.
S'ils reviennent aux nasses à doubles compartiments, en 1986, Bertrand et Richard proposent une première méthodologie pour le choix des sites de piégeage. Il semble plus efficace sur des cours d'eau larges de 2 à 10 mètres à débit moyen ou fort. Les périodes d'étiage les plus sévères sont à éviter. Enfin, les berges avec un chevelu racinaire dense sont plus intéressantes (Bertrand \& Richard 1986). Un taux de capture jugé trop faible, une moyenne d'un desman capturé pour plus de 20 nuits-pièges, incite Bertrand à développer prioritairement la détection via la recherche de fèces (Bertrand 1994). Cette méthode devient par la suite la plus utilisée pour mettre en évidence la présence de l'espèce (Queiroz et al. 1996; Aymerich et al. 2001; Charbonnel et al. 2014; Biffi et al. 2017; Aymerich 2018) bien que les captures ne soient pas abandonnées pour autant. La complémentarité des deux méthodes est étudiée en 2003 sur 26 réplicas (González-Esteban et al. 2003). Ils disposent entre 6 et 21 nasses à fil métallique, contrôlées toutes les trois heures pendant une à trois nuits, sur 16 rivières du nord de l'Espagne et sur des linéaires variables de 800 à 3000 mètres. Ce protocole est complété par deux passages de recherche de fèces sauf si le résultat est positif dès la première fois. Les résultats montrent une réelle plus-value des captures avec 11 réplicas positifs à la capture, négatifs aux feces, aucun dans le sens inverse. Cinq réplicas sont positifs aux deux méthodes. Le taux de capture sur cette étude est de 16,1\%.

En 2010, Bertrand utilise des nasses à double compartiment pour compléter des prospections fèces (Bertrand 2010). Entre 2002 et 2009, Melero utilise le piégeage pour capturer et équiper d'un transpondeur et/ou d'un émetteur, 45 individus sur la rivière Tor, dans l'est des Pyrénées espagnoles. Le type de nasse n'est pas précisé mais le taux de capture est proche de $10 \%$ (Melero et al. 2012). En 2018, pour une autre opération de radiopistage au pays basque espagnol, 31 desmans sont capturés pour un taux de capture de $25 \%$ (Esnaola et al. 2018). Elle reprend le même protocole de piégeage que Gonzalez-Esteban et al. (2003).

Sur la même période, entre 2011 et 2018,80 opérations de captures ont été réalisées dans les Pyrénées françaises. Le Desman étant une espèce protégée, toutes ces opérations ont été menées sous la responsabilité d'au moins une personne disposant d'une autorisation préfectorale pour la capture et le relâcher de spécimens. Nous proposons ici un bilan et un retour d'expériences sur l'ensemble de ce travail. L'objectif est aussi de proposer des éléments de protocole pour optimiser les probabilités de capture de Desman des Pyrénées et de proposer une analyse similaire aux travaux précités de Gonzalez-Esteban et al. (2003) sur le comparatif entre les captures et la recherche de crottes.

\section{MATÉRIELS ET MÉTHODES}

\section{LOCALISATION DE L'ÉTUDE}

Les 80 opérations ont été réalisées sur toute la chaîne des Pyrénées françaises, réparties sur 44 stations pour 24 cours d'eau ou lacs différents (Tableau 1). 
TABLEAU 1. - Liste des sites d'opérations de capture. Abréviations: Dép., département; N2000, Natura 2000.

\begin{tabular}{|c|c|c|c|c|c|c|}
\hline Nom (Rivière, Station) & N2000 & Dép. & $\begin{array}{l}\text { Nombre } \\
\text { d'opération(s) }\end{array}$ & Année(s) & Altitude & $\begin{array}{l}\text { Site } \\
\text { limite }\end{array}$ \\
\hline Gave de Saint-Engrâce, camping & FR7200790 & 64 & 1 & 2014 & $389 \mathrm{~m}$ & - \\
\hline Gave de Saint-Engrâce, église & FR7200790 & 64 & 1 & 2014 & $567 \mathrm{~m}$ & - \\
\hline Gave d'Ossau, Soussouéou & FR7200793 & 64 & 1 & 2015 & $952 \mathrm{~m}$ & - \\
\hline Gave de Bious, amont du lac de Bious & FR7200793 & 64 & 1 & 2015 & $1421 \mathrm{~m}$ & - \\
\hline Gave de Bious, plateau de Bious & FR7200793 & 64 & 2 & 2015,2017 & $1546 \mathrm{~m}$ & - \\
\hline Gave de Bious, lac d'Ayous & FR7200793 & 64 & 1 & 2014 & $2058 \mathrm{~m}$ & - \\
\hline Gave de Pau, cirque de Gavarnie & FR7300927 & 65 & 1 & 2015 & $1543 \mathrm{~m}$ & oui \\
\hline Gave d'Estaubé, cirque d'Estaubé & FR7300927 & 65 & 1 & 2014 & $1689 \mathrm{~m}$ & oui \\
\hline Ruisseau du Maillet, Troumouse & FR7300927 & 65 & 1 & 2016 & $1670 \mathrm{~m}$ & oui \\
\hline Ruisseau du Cot, cirque de Troumouse & FR7300927 & 65 & 1 & 2015 & $2063 \mathrm{~m}$ & oui \\
\hline Rioumajou, pont Tisné & FR7300934 & 65 & 1 & 2017 & $1144 \mathrm{~m}$ & oui \\
\hline Rioumajou, amont barrage & FR7300934 & 65 & 1 & 2014 & $1368 \mathrm{~m}$ & oui \\
\hline Rioumajou, Frédancon & FR7300934 & 65 & 1 & 2015 & $1393 \mathrm{~m}$ & oui \\
\hline Rioumajou, Barguerette & FR7300934 & 65 & 1 & 2016 & $1493 \mathrm{~m}$ & oui \\
\hline Pique, Salles-et-Pratviel & FR7301822 & 9 & 1 & 2016 & $605 \mathrm{~m}$ & oui \\
\hline Salat, aval Lacourt station 2 & FR7301822 & 9 & 1 & 2015 & $409 \mathrm{~m}$ & oui \\
\hline Salat, aval Lacourt station 1 & FR7301822 & 9 & 1 & 2015 & $414 \mathrm{~m}$ & oui \\
\hline Salat, aval Kercabanac & FR7301822 & 9 & 1 & 2014 & $437 \mathrm{~m}$ & oui \\
\hline Salat, pont de la Taule & FR7301822 & 9 & 1 & 2016 & $596 \mathrm{~m}$ & - \\
\hline Arize, Estaniels & - & 9 & 2 & 2013 & $489 \mathrm{~m}$ & - \\
\hline Artignac, Castelnau-Durban & - & 9 & 1 & 2013 & $368 \mathrm{~m}$ & - \\
\hline Vicdessos, pont d'Illier* & - & 9 & 2 (radiotrack) & 2018 & $643 \mathrm{~m}$ & - \\
\hline Vicdessos, pont d'Arconac aval & - & 9 & 2 (radiotrack) & 2018 & $679 \mathrm{~m}$ & - \\
\hline Vicdessos, pont d'Arconac amont & - & 9 & 2 (radiotrack) & 2018 & $685 \mathrm{~m}$ & - \\
\hline Ariège, Vèbre/Urs & FR7301822 & 9 & 1 & 2016 & $558 \mathrm{~m}$ & - \\
\hline Aston, confluence des Ubals & FR7300827 & 9 & 15 (radiotrack) & 2014,2015 & $590 \mathrm{~m}$ & - \\
\hline Aston, grange de Sigueille & FR7300827 & 9 & 2 (radiotrack) & 2014 & $730 \mathrm{~m}$ & - \\
\hline Aston, confluence Sirbal & FR7300827 & 9 & 1 (radiotrack) & 2014 & $757 \mathrm{~m}$ & - \\
\hline Aston, zone Aston amont, Sirbal & FR7300827 & 9 & 3 (radiotrack) & 2015 & $781 \mathrm{~m}$ & - \\
\hline Aston, Pla de las Peyres & FR7300827 & 9 & 1 & 2014 & $1700 \mathrm{~m}$ & - \\
\hline Hers, L'Aiguillon & FR7301822 & 9 & 2 & 2014,2015 & $466 \mathrm{~m}$ & oui \\
\hline Hers, le Raspier & FR7301822 & 9 & 1 & 2015 & $515 \mathrm{~m}$ & oui \\
\hline Rebenty, Joucou & FR9101468 & 11 & 1 & 2014 & $609 \mathrm{~m}$ & oui \\
\hline Rebenty, secteur pont du Roi & FR9101468 & 11 & 1 & 2015 & $693 \mathrm{~m}$ & oui \\
\hline Rebenty, Bec d'Argent & FR9101468 & 11 & 1 & 2016 & $1238 \mathrm{~m}$ & oui \\
\hline Restanque - Bruyante, Artigues & FR7300831 & 9 & 1 & 2015 & $1615 \mathrm{~m}$ & - \\
\hline Étang de Cailloung, Fa d'en Marra & FR7300831 & 9 & 1 & 2015 & $1483 \mathrm{~m}$ & - \\
\hline Lac de Quérigut, Quérigut & FR7300831 & 9 & 1 & 2016 & $1876 \mathrm{~m}$ & - \\
\hline Aude, station des Pneus & FR9101470 & 11 & 7 (radiotrack) & 2011, 2012, 2013 & $495 \mathrm{~m}$ & - \\
\hline Aude, passerelle de Gesse & FR9101470 & 11 & 6 (radiotrack) & $2011,2012,2013$ & $542 \mathrm{~m}$ & - \\
\hline Aude, Escouloubre & FR9101470 & 11 & 3 (radiotrack) & 2011,2013 & $864 \mathrm{~m}$ & - \\
\hline L'Aiguette, Counozouls & FR9101470 & 11 & 1 & 2017 & $1309 \mathrm{~m}$ & - \\
\hline Clot, étang du Clot & FR9101473 & 66 & 1 & 2015 & $1661 \mathrm{~m}$ & - \\
\hline Étang de l'Estelat & FR9101473 & 66 & 1 & 2015 & $2021 \mathrm{~m}$ & - \\
\hline
\end{tabular}

Parmi ces 80 opérations, 18 ont été réalisées sur 17 stations qualifiées par la suite de «sites limites». C'est une notion arbitraire qui correspond à des sites où la présence de l'espèce est fortement remise en question pour diverses raisons. Ce sont soit des sites en limite de répartition du Desman, soit des sites sur lesquels des problèmes ayant entraîné une disparition du Desman ont été constatés. C'est le cas pour les quatre opérations du site FR7300934 et les trois du site FR9101468 (pollutions, perturbations hydrauliques successives, etc.). Pour ne pas biaiser les résultats, ces opérations seront dans un second temps retirées du jeu de données pour certaines analyses.

\section{PréSENTATION DU MATÉRIEL DE PIÉGEAGE}

Entre 2011 et 2018, plusieurs modèles de nasses ont été utilisés en s'inspirant des différentes évolutions détaillées dans la revue historique proposée en introduction. La Fig. 1 présente ces différents modèles.
Des nasses doubles (Fig. 1) sont utilisées entre 2011 et 2014 lors de 29 opérations. Il s'agit d'un dispositif constitué de deux nasses à anguilles assemblées et disposant chacune d'une empêche de $40 \mathrm{~mm}$. Le treillis est en fil souple avec un maillage carré de $20 \mathrm{~mm}$. Des arceaux et une entrée rigide permettent de maintenir le piège et de le rigidifier pour le rendre plus résistant dans le courant. Des tiges métalliques latérales peuvent aussi être ajoutées. L'entrée du piège doit être immergée d'au moins $50 \%$ et le second compartiment être maintenu hors de l'eau. Ces modèles sont abandonnés et remplacés peu à peu par des nasses simples à partir de 2014.

Les autres modèles utilisés n'ont qu'une seule nasse, bien que la seconde permettait de renforcer la difficulté pour un Desman capturé de ressortir du piège. Cette modification permet d'avoir des pièges plus légers, moins encombrants et donc plus facilement transportables en plus grand nombre. 


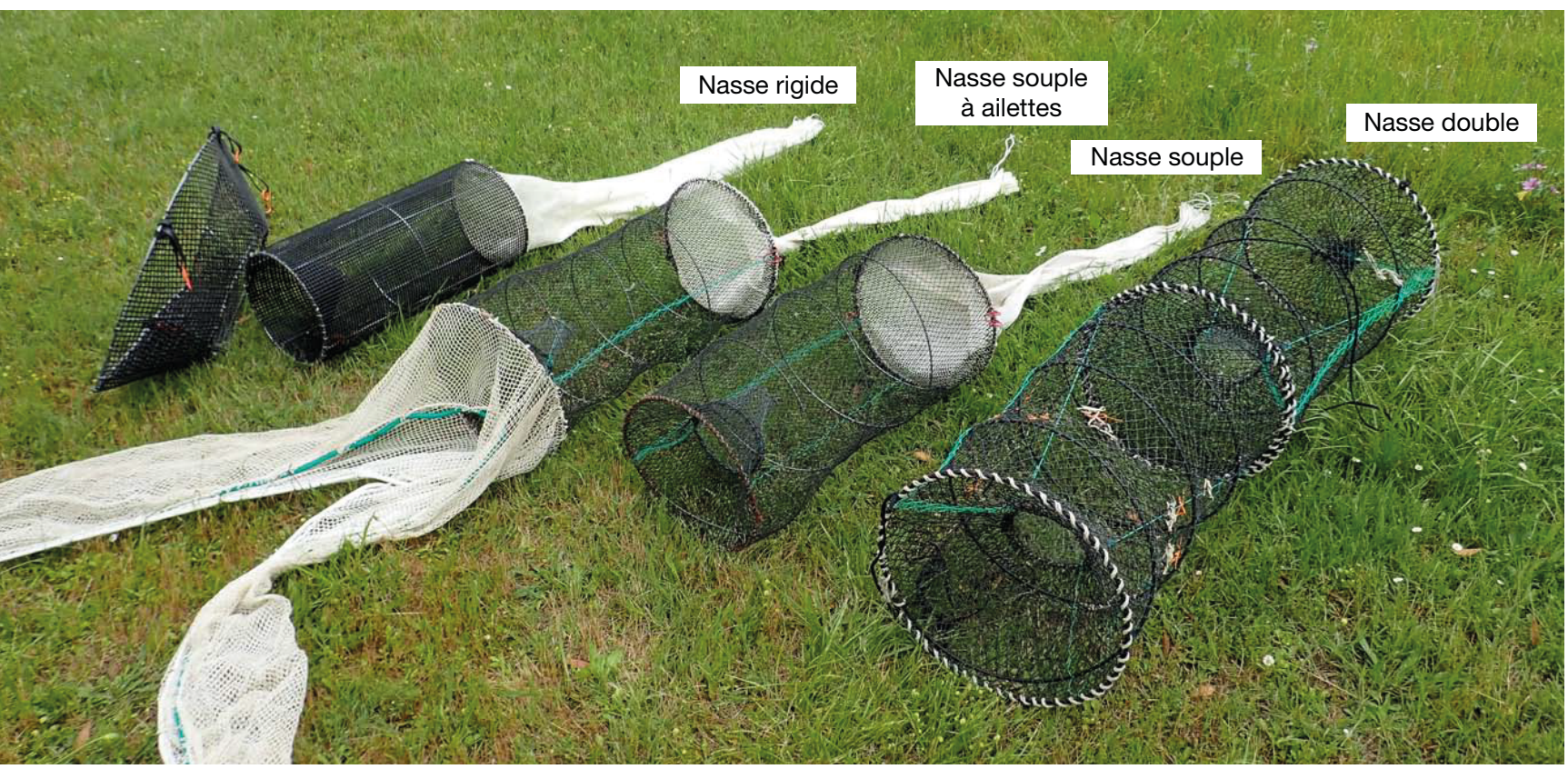

FIG. 1. - Modèles de nasses utilisées. Crédit photo: Mélanie Némoz - CEN Occitanie.

Ils sont en revanche équipés d'un filet à maille resserrée de $6 \mathrm{~mm}$ permettant la mise au sec appelé "chaussette».

Des nasses souples (Fig. 1) sont utilisées entre 2014 et 2019. Ces nasses présentent une empêche de $60 \mathrm{~mm}$ et un treillis en fil souple à maillage carré de $20 \mathrm{~mm}$. Un cas de mortalité a été constaté en 2014 lorsqu'un desman s'est coincé la tête dans une maille et s'est noyé. Après diverses mesures réalisées sur des spécimens de desmans analysés dans le cadre de la valorisation des cadavres (Fournier-Chambrillon et al. 2019), le maillage du treillis a été réduit à $12 \mathrm{~mm}$. Toutes les nasses ont été modifiées en ce sens.

Des échanges sur le terrain avec l'équipe ARCEA en Galice ont inspiré l'idée d'équiper certaines nasses souples à ailettes (Fig. 1). Ce sont des filets de trois à cinq mètres accrochés de part et d'autre de l'entrée de la nasse. Ils permettent de barrer tout ou partie d'un cours d'eau et donc de canaliser physiquement les desmans vers le piège. Elles sont utilisées entre 2013 et 2018.

Des nasses rigides (Fig. 1) sont utilisées entre 2014 et 2018 et ont été conçues avec la deuxième génération des nasses simples souples. Le treillis n'est plus composé de fils mais d'un plastique rigidifié avec un maillage carré de $10 \mathrm{~mm}$. Cette matière donne une plus grande résistance aux pièges qui peuvent alors être positionnés dans des zones de plus forts courants. Elles peuvent aussi être équipées d'une «tête» qui permet d'augmenter la surface d'entrée dans la nasse, ce qui est utile dans les veines de courant un peu plus larges.

Depuis 2014, les types nasses souples, à ailettes et rigides sont utilisés simultanément. Il est donc possible d'apporter des éléments de comparaison sur l'efficacité de ces différents modèles.

Enfin, un cinquième type de nasse a été utilisé de façon marginale lors de trois opérations de 2013. C'est un modèle qu'utilisaient alors des chercheurs espagnols (Julio Gisbert, comm. pers.) et qui s'apparente globalement au modèle de la nasse simple souple sans la présence de la chaussette et d'une taille un peu plus petite.

\section{PROTOCOLE DE PIÉGEAGE}

Lors des 80 opérations menées en huit ans, $28 \pm 12$ pièges ( $\mu=28,04$ et $\sigma=12,24)$ sont installés sur les secteurs de piégeage. Le nombre varie selon les conditions du tronçon et le matériel disponible (Fig. 2A). Elles ne sont pas appâtées, ce sont des pièges comportementaux. Le Desman recherche sa nourriture dans l'eau, généralement en évoluant dans les veines de courant de l'aval vers l'amont. Aussi, les équipes de piégeage utilisent cette caractéristique en plaçant les pièges directement dans les voies d'eau, rarement dans les zones calmes, l'entrée orientée vers l'aval et immergée. Les desmans peuvent malgré tout être "guidés" vers les nasses par la construction de petits barrages en pierre ou par l'utilisation des ailettes. En se déplaçant, ils sont donc susceptibles de pénétrer dans les nasses d'où ils ne peuvent ressortir grâce au système de l'empêche.

Selon les secteurs, le linéaire de piégeage n'est pas homogène (Fig. 2B). Il correspond à la longueur de cours d'eau entre le piège le plus à l'aval et le piège le plus en amont. Cette diversité s'explique par la volonté de tester deux hypothèses pour optimiser les chances de capture et de détection de l'espèce, concentrer les pièges sur un seul domaine vital théorique (linéaire inférieur ou égal à $500 \mathrm{~m}$ ) ou essayer d'en couvrir davantage en augmentant le linéaire de piégeage. Suite à de récentes études de radiopistage (Melero et al. 2012; Lim \& Jacob 2019), ce domaine vital théorique est estimé à $500 \mathrm{~m}$ pour un desman sur une courte durée de suivi allant de quelques jours à trois semaines. Pour l'analyse, le linéaire de piégeage sera donc classé en deux catégories, inférieur ou supérieur à $500 \mathrm{~m}$. Dans tous les cas, sur un tronçon de 
A

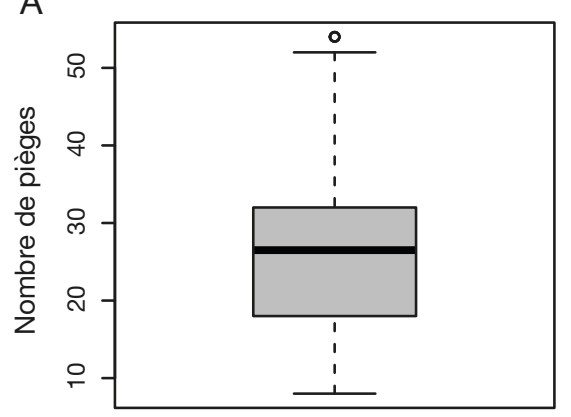

B

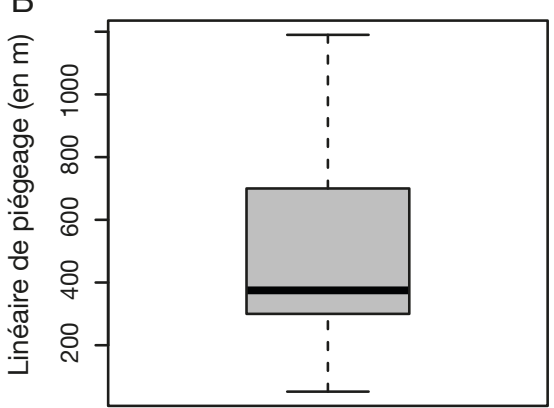

C

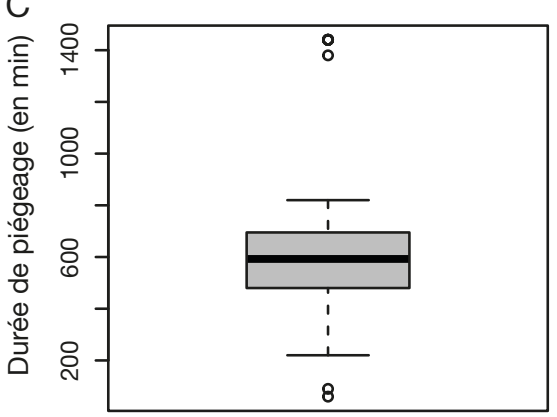

FIG. 2. - Répartition des variables nombre de pièges (A), linéaire de piégeage (B) et durée de piégeage (C).

TABLEAU 2. - Répartition des opérations en fonction du nombre de captures et du nombre d'individus capturés.

\begin{tabular}{|c|c|c|c|c|c|c|}
\hline \multirow{2}{*}{$\begin{array}{r}\text { Nombre de } \\
\text { captures }\end{array}$} & \multicolumn{6}{|c|}{ Nombre d'individus capturés } \\
\hline & Pas de captures & 1 Individu & 2 individus & 3 individus & 4 individus & Inconnu \\
\hline 0 & 44 & - & - & - & - & - \\
\hline 1 & - & 24 & - & - & - & - \\
\hline 2 & - & 1 & 5 & - & - & 1 \\
\hline 3 & - & - & - & 1 & - & 1 \\
\hline 4 & - & - & - & 1 & 1 & - \\
\hline 5 & - & - & - & - & 1 & - \\
\hline
\end{tabular}

rivière donné, le secteur de piégeage est choisi de façon à correspondre autant que possible à l'optimum décrit en 1986 (Bertrand \& Richard 1986). Les zones riches en affluents ou en annexes hydrauliques sont également privilégiées.

Aucune mention n'est faite dans la bibliographie d'une tentative de capture de desmans dans un milieu lacustre. Ce n'est qu'en 2015 que cela a été tenté. Les nasses ont alors été positionnées le long de la berge, de manière parallèle ou avec un angle plus ou moins ouvert, dans la végétation ou contre des blocs rocheux en fonction de la configuration du lac.

Sauf protocoles particuliers ou conditions défavorables, les nasses sont installées dans l'après-midi, laissées en place pendant toute la nuit et retirées le lendemain matin au lever du jour. Pour limiter au maximum les risques pour le Desman, un relevé est prévu toutes les 1 h30 au maximum, soit environ une heure entre le contrôle du dernier piège et le début du relevé suivant. La durée de piégeage n'est pas restée fixe durant toute la période étudiée (Fig. 2C). Entre 2011 et 2013, les opérations étaient stoppées en milieu de nuit. À partir de 2014, elles sont maintenues jusqu'à l'aube dans la mesure du possible. Enfin, pour les opérations avec un objectif de radiopistage, les nasses peuvent être laissées en permanence $(24 \mathrm{~h} / 24)$. Cette variabilité ne permet pas de parler de nuits-pièges pour tout le jeu de données ni de comparer les résultats sur ce pas de temps. L'échelle prise en compte est donc l'heure et des taux horaire de capture seront proposés dans les différentes analyses en se basant sur la notion d' "heure-piège». Une heure-piège correspond à un piège posé pendant une heure. Ainsi, sur la période considérée, la pression de piégeage s'élève à 2243 nuits-pièges ou 25965,41 heures-pièges.
Toutefois, si elle n'est pas utilisée dans l'analyse statistique, la notion de taux de capture par rapport à un nombre de nuitspièges demeure présente au moins à titre indicatif et surtout pour avoir un élément de comparaison avec les autres études dont il est question précédemment. C'est l'unité généralement utilisée par la plupart des auteurs.

Enfin, lors de 35 des 80 opérations, une prospection fèces a été effectuée sur tout le linéaire du secteur de piégeage afin de tester la complémentarité des deux méthodes.

\section{PRÉSENTATION DES RÉSULTATS}

\section{NOMBRE DE CAPTURES}

57 captures ont été faites sur 80 opérations soit $0,71 \pm 1,04$ capture par opération. Elles sont réparties sur 36 opérations positives pour un maximum de cinq captures sur une seule opération. À l'inverse notamment de Puisségur, jamais deux desmans n'ont été capturés simultanément dans un même piège. Au cours de ces 36 opérations positives, il a généralement été possible de déterminer précisément le nombre d'individus capturés grâce à des marquages ou à des méthodes génétiques. La relation entre ces deux variables est présentée dans le Tableau 2.

\section{RÉPARTITION GÉOGRAPHIQUE ET TAUX DE CAPTURE}

La Figure 3 présente la localisation de tous les secteurs ainsi que la capture ou non d'au moins un Desman sur chacun d'entre eux.

La répartition géographique est déséquilibrée entre les différents sites Natura 2000 concernés. Les sites avec le plus d'opérations sont FR7300827 puis FR9101470, sur 


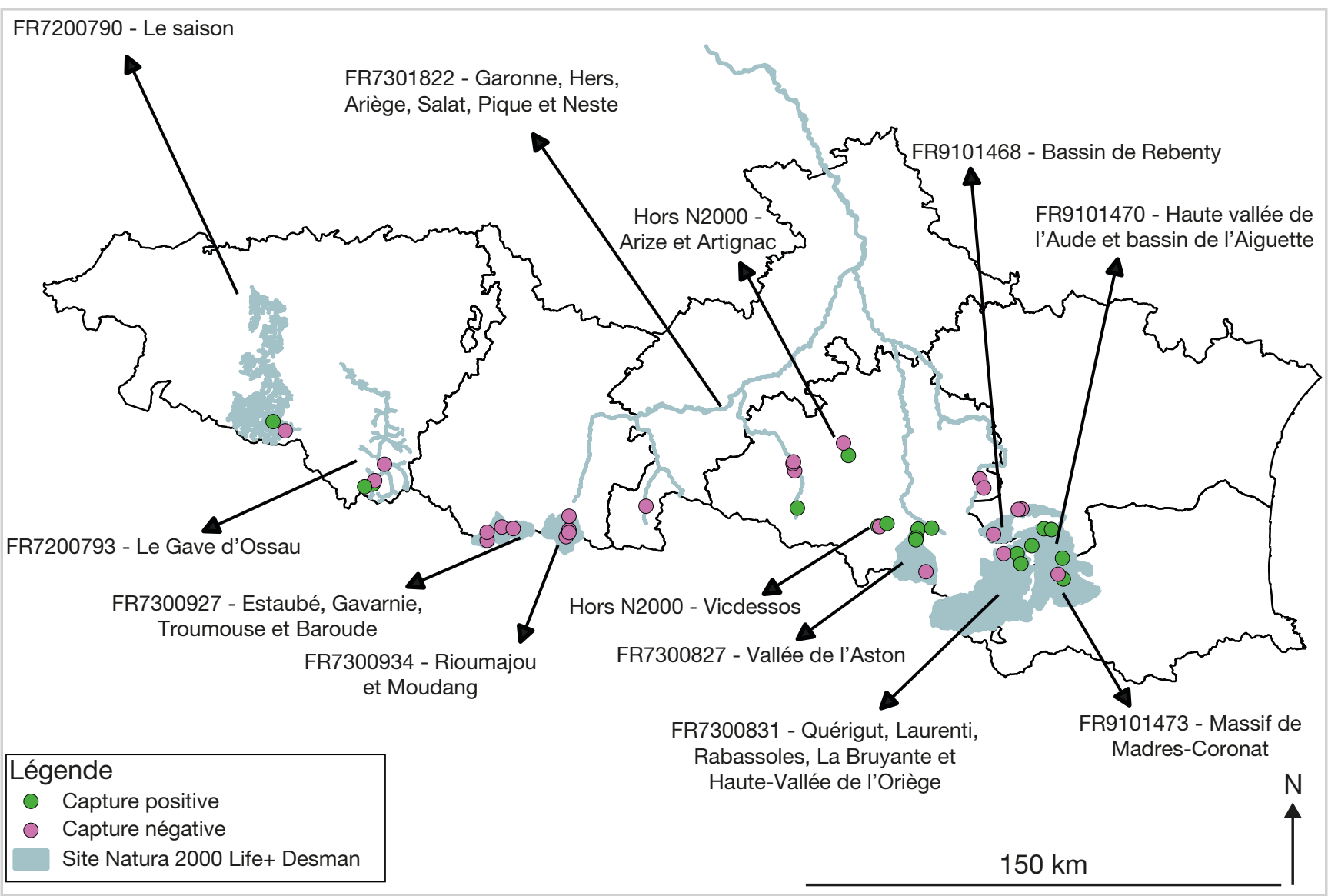

FIG. 3. - Localisation des secteurs et résultats associés. Cartographie: É. Poncet, CEN MP 2019 - Traitement QGIS 2.18.13. Source des données: Life+ Desman (2011-2018).

lesquels des études demandant une forte pression de piégeage ont été menées (respectivement un suivi par radiopistage et une opération de capture-marquage-recapture pour étudier l'impact de l'augmentation des débits sur les populations de desmans). Cette pression de capture plus importante est mise en évidence dans le Tableau 3 avec respectivement 6531,33 et 2591,25 heures-pièges. La plus forte pression a été mise sur la catégorie " Hors sites N2000" qui contient la rivière Vicdessos où une opération de radiopistage a également été menée afin d'étudier la sélection des habitats par le Desman et la sensibilité de ceux-ci aux variations de débit.

Le taux de capture global est de 2,54\% (57 desmans pour 2243 nuits-pièges), cette valeur remonte à 3,05\% en retirant les sites limites (57 desmans pour 1868 nuits-pièges). Le Tableau 3 présente le détail pour chaque site. Toutefois, pour la suite des analyses nous parlerons plutôt de taux horaire de capture. Globalement celui-ci est de 2,20 \%o (57 desmans capturés en 25965,41 heures-pièges), de $2,56 \%$ en retirant les sites limites ( 57 desmans en 22253,44 heures-pièges).

On observe cependant une forte hétérogénéité entre les sites. Localement, ces deux indicateurs sont bien supérieurs aux valeurs globales sur les sites FR7300831, FR9101470 et FR7200793. À l'inverse, aucun Desman n'a été capturé sur certains sites.
Protocole DE PIÉGEAGE

Le protocole de piégeage pour une opération est déterminé par trois variables: le nombre de pièges posés, le linéaire de piégeage et la durée du piégeage. Les coefficients de corrélation entre le nombre de captures et ces trois variables sont respectivement de $-0,03 ; 0,25$ et $-0,09$. Aucune de ces corrélations n'apparaît significative et c'est toujours le cas en retirant les sites limites du jeu de données (respectivement de $-0,04 ; 0,06$ et $-0,14)$.

Par rapport à l'objectif de vérifier si le nombre de captures était plus élevé sur des linéaires inférieurs ou supérieurs à $500 \mathrm{~m}$, un test du chi ${ }^{2}$ supplémentaire a été effectué. Pour les 62 opérations où le linéaire a été relevé, la répartition du nombre d'heures-pièges est présentée dans le Tableau 4.

Le test montre une différence significative en faveur du nombre de capture sur des linéaires supérieurs à $500 \mathrm{~m}$ ( $\mathrm{chi}^{2}$ mesuré $=$ $29,12>\mathrm{chi}^{2}$ théorique $=10,82$ pour $\mathrm{p}$-value $=0,001, \mathrm{ddl}=1$ ) .

\section{HORAIRE DES CAPTURES}

Pour chaque capture d'un Desman des Pyrénées, l'horaire à laquelle la capture est constatée a systématiquement été relevé, ce qui permet d'avoir un jeu de données complet. Notons que cette valeur ne correspond pas exactement à l'horaire à laquelle un Desman est effectivement piégé, celui-ci n'étant pas connu car la capture peut intervenir à tout moment entre 
TABLEAU 3. - Bilan des opérations par site Natura 2000.

\begin{tabular}{|c|c|c|c|c|c|c|c|}
\hline Site & $\mathbf{N}$ opérations & $\begin{array}{l}\text { Taux } \\
\text { d'opérations } \\
\text { positives }\end{array}$ & $\mathrm{N}$ captures & $\begin{array}{c}\mathrm{N} \text { heures- } \\
\text { pièges }\end{array}$ & $\begin{array}{l}\text { Taux horaire de } \\
\text { capture }\end{array}$ & $\mathrm{N}$ nuits-pièges & Taux de capture \\
\hline FR 7200790 & 2 & $50 \%$ & 1 & 667 & $1,50 \%$ & 58 & $1,72 \%$ \\
\hline FR 7200793 & 5 & $60 \%$ & 8 & 1236,83 & $6,47 \%$ & 123 & $6,50 \%$ \\
\hline FR 7300927 & 4 & $0 \%$ & 0 & 852 & $0,00 \%$ & 100 & $0 \%$ \\
\hline FR 7300934 & 4 & $0 \%$ & 0 & 1014,25 & $0,00 \%$ & 107 & $0 \%$ \\
\hline FR 7301822 & 9 & $22,20 \%$ & 6 & 2095,5 & $2,86 \%$ & 209 & $2,87 \%$ \\
\hline FR 7300827 & 22 & $41 \%$ & 10 & 6531,33 & $1,53 \%$ & 579 & $1,72 \%$ \\
\hline FR 7300831 & 3 & $66,70 \%$ & 5 & 529,5 & $9,44 \%$ & 81 & $6,17 \%$ \\
\hline FR 9101468 & 3 & $0 \%$ & 0 & 961,75 & $0,00 \%$ & 92 & $0 \%$ \\
\hline FR 9101470 & 17 & $70,60 \%$ & 18 & 2591,25 & 6,95 \%。 & 370 & $4,86 \%$ \\
\hline FR 9101473 & 2 & $50 \%$ & 2 & 545 & $3,67 \%$ & 50 & $4 \%$ \\
\hline Hors sites & 9 & $66,70 \%$ & 7 & 8941 & $0,78 \%$ & 474 & $1,48 \%$ \\
\hline Bilan & 80 & $45 \%$ & 57 & 25965,41 & 2,20 \%。 & 2243 & $2,54 \%$ \\
\hline
\end{tabular}

TABLEAU 4. - Répartition des heures-pièges selon la classe de linéaire.

\begin{tabular}{lrcc}
\cline { 2 - 4 } & \multicolumn{2}{c}{} & \multicolumn{2}{l}{$\begin{array}{l}\text { Nombre } \\
\text { d'heures- } \\
\text { pièges total }\end{array}$} \\
\hline Linéaire $<=500 \mathrm{~m}$ & 16516,83 & 26 & 16542,83 \\
Linéaire $>500 \mathrm{~m}$ & 3512,58 & 23 & 3535,58 \\
\hline Total & 20029,41 & 49 & 20078,41 \\
\hline
\end{tabular}

deux relevés. Pour rappel, la durée maximale entre deux contrôles est d'une heure et demie. Néanmoins, la Figure 4 montre que des captures peuvent intervenir à tout moment de la nuit avec deux pics un peu plus marqués entre $22 \mathrm{~h}$ et $23 \mathrm{~h} 30$, puis entre $03 \mathrm{~h} 30$ et $06 \mathrm{~h} 00$. Ces deux pics peuvent être légèrement décalés dans le temps en tenant compte de la remarque précédente quant au décalage entre l'horaire réel mais inconnu d'une capture et l'horaire de sa constatation. Par ailleurs, la répartition horaire des captures est potentiellement biaisée car la durée des opérations de captures n'est pas homogène. La Figure 4 montre donc aussi la pression de capture totale pour chaque tranche demi-horaire. Il y a une corrélation positive entre ces deux variables (coefficient de corrélation de Spearman $=0,42$ ) mais qui n'est pas pour autant significative.

Bien que cela ne représente qu'un faible volume de l'effort de capture global (total de 4111,83 heures-pièges entre $8 \mathrm{~h}$ et $18 \mathrm{~h}$ soit $15,84 \%$ du volume total), dans plusieurs cas, les opérations de capture se sont poursuivies sur la journée. Les captures de deux desmans ont ainsi été constatées à $12 \mathrm{~h} 30$ et à $17 \mathrm{~h} 56$.

Enfin, la Figure 5 présente l'horaire de la première capture pour chaque opération. Celle-ci peut intervenir à tout moment de la nuit, y compris à l'aube. Sur les 36 opérations positives, 16 ont eu une première capture après minuit et quatre d'entre elles après $7 \mathrm{~h}$.

\section{EFFICACITÉ DES PIÈGES}

Le Tableau 5 présente le bilan obtenu pour chaque type de pièges. Il est intéressant de remarquer que tous les types de nasses ont permis de capturer au moins un Desman. Les nasses souples simples sont celles qui ont permis le plus de captures mais ce sont aussi les pièges qui ont été les plus utilisés depuis 2011. Le rapport de ces deux variables montre que le taux de capture horaire est plus élevé pour les nasses souples à ailettes puis pour les doubles nasses.

Les doubles nasses, abandonnées en 2014, n’ont que peu été utilisées avec les trois autres modèles utilisés depuis. Elles ne sont donc pas prises en compte dans l'analyse suivante. Entre ces trois types de nasses et sur la totalité des opérations où elles sont utilisées simultanément (28 opérations depuis 2014), le test du chi ${ }^{2}$ démontre une hétérogénéité significative des taux de capture $\left(\mathrm{chi}^{2}\right.$ mesuré $=8,84>\mathrm{chi}^{2}$ théorique $=7,82$ pour $\mathrm{p}$-value $=0,05, \mathrm{ddl}=2$ ). Les nasses souples à ailettes ont un taux de capture horaire significativement supérieur aux nasses simples souples ou rigides. C'est toujours le cas en retirant les sites limites du jeu de données, soit neuf opérations sur les 28 exploitées précedemment $\left(\mathrm{chi}^{2}\right.$ mesuré $=9,4>\mathrm{chi}^{2}$ théorique $=9,21$ pour $\mathrm{p}$-value $=0,01, \mathrm{ddl}=2$ ).

\section{INFLUENCE DU MILIEU}

Des opérations de captures ont été menées dans deux grands types d'habitats, les cours d'eau et les milieux lacustres. Le Tableau 6 présente le bilan des taux horaires de capture pour ces deux catégories. Le test du chi ${ }^{2}$ révèle une différence $\left(\mathrm{chi}^{2}\right.$ mesuré $=$ $6,47>\mathrm{chi}^{2}$ théorique $=5,41$ pour $\mathrm{p}$-value $=0,02, \mathrm{ddl}=1$ ) avec un taux de capture horaire significativement plus élevé dans les milieux lacustres que sur les cours d'eau. Bien que tous les sites limites soient sur des cours d'eau, cette différence demeure en les retirant du jeu de données $\left(\mathrm{chi}^{2}\right.$ mesuré $=4,48>\mathrm{chi}^{2}$ théorique $=3,84$ pour $\mathrm{p}$-value $=0,05, \mathrm{ddl}=1$ ) .

\section{RELATION CAPTURE/ PROSPECTION FÈCES}

Le Tableau 7 présente les résultats des 35 opérations sur lesquelles une prospection fèces a été menée en complément de la pose de nasses. Au total, huit opérations sont positives aux deux méthodes, quatre sont positives aux fèces et négatives à la capture tandis que deux suivent le schéma inverse. 21 opérations sont négatives aux deux méthodes. Le test du chi ${ }^{2}$ montre une différence significative entre ces valeurs observées et les valeurs théoriques ( $\mathrm{chi}^{2}$ mesuré $=12,99>\mathrm{chi}^{2}$ théorique $=10,82$ pour $\mathrm{p}$-value $=0,001$ et $\mathrm{ddl}=1$ ). Glo- 


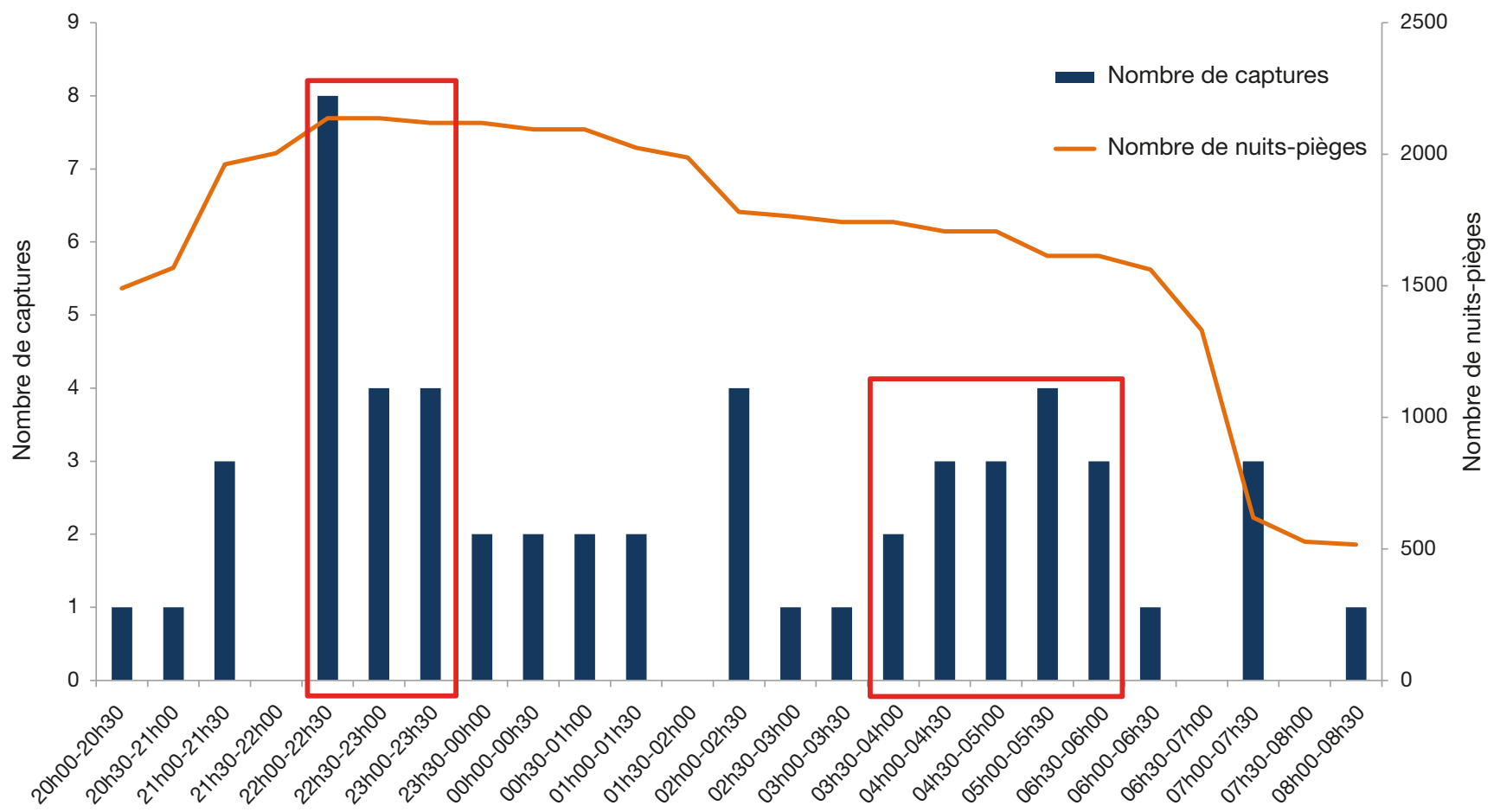

FIG. 4. - Répartition du nombre de captures constatées par tranche demi-horaire en fonction du nombre de nuits-pièges.

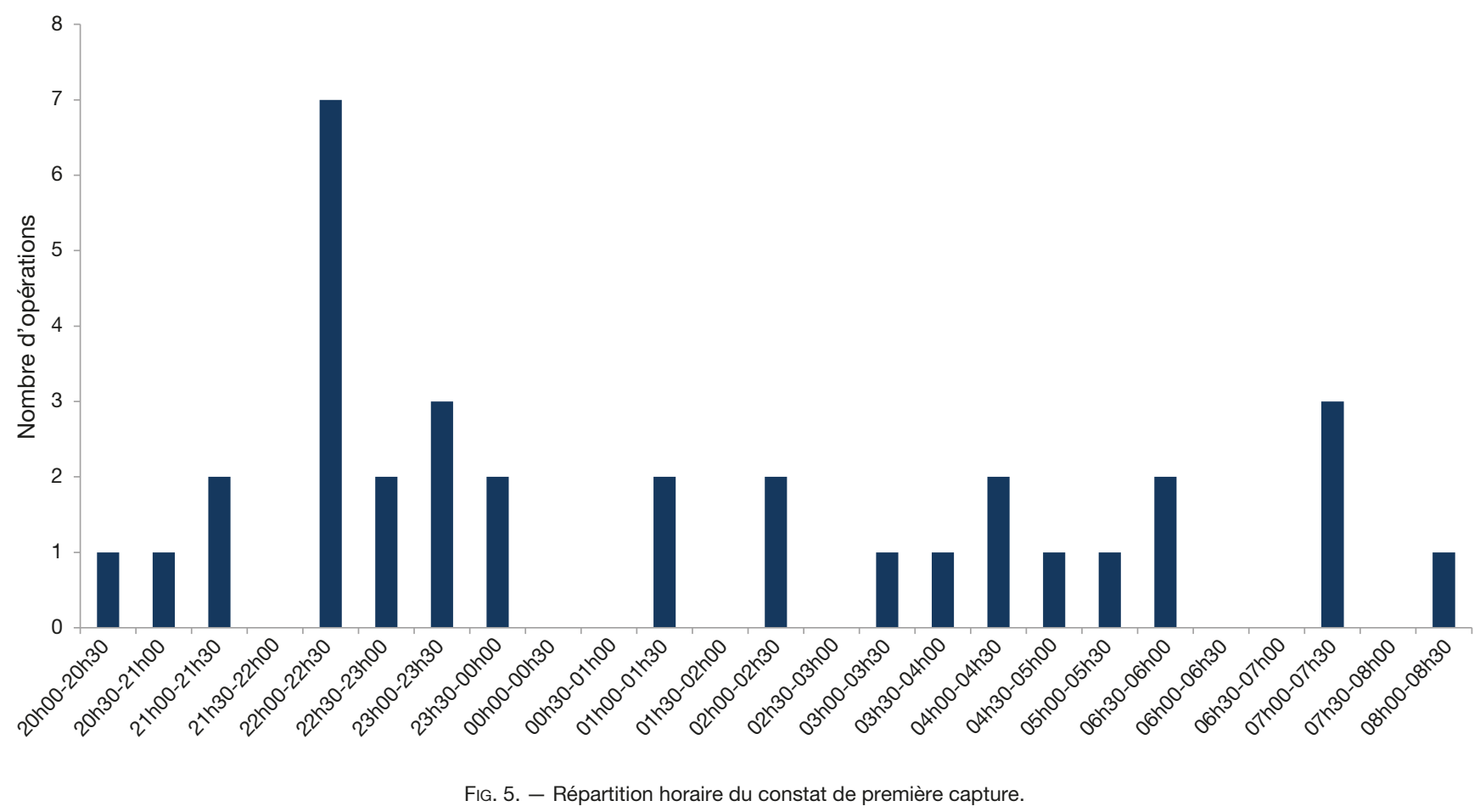

balement, ces résultats montrent donc un déséquilibre des résultats obtenus en faveur des opérations de type +/+ et -/-. Cela tend à indiquer l'absence d'une plus-value des captures par rapport à la recherche de fèces.

Outre ce résultat global, il est intéressant de signaler que les deux opérations positives à la capture et négatives aux feces ont toutes deux été réalisés sur des milieux lacustres.

\section{DISCUSSION}

Le taux de capture global (2,54\%) obtenu en France depuis 2011 parait faible au regard des chiffres avancés lors de travaux antérieurs ou d'études contemporaines en Espagne ou au Portugal. Les raisons possibles de cette différence sont la très grande hétérogénéité de nos secteurs de captures et les 
TABLEAU 5. - Bilan de l'efficacité pour chaque type de piège. *, le nombre d'heures-pièges présenté ici ne correspond pas à la valeur totale énoncée précedemment puisque ces données ne tiennent pas compte d'une partie du piégeage intensif mené sur l'Aston pour une opération de radiopistage. Le détail des nasses n'y a pas toujours été relevé. Les desmans capturés lorsque les détails des nasses ne sont pas répertoriés ne sont pas non plus pris en compte. C'est la raison pour laquelle seulement 52 desmans apparaissent au lieu des 57 annoncés.

\begin{tabular}{lccc}
\hline Type de nasses & Nb heures-pièges & Nb captures & Taux horaire de captures \\
\hline Nasses « espagnoles» & 975 & 1 & $1,03 \% \circ$ \\
Doubles nasses & 4227,25 & 18 & $4,26 \% \circ$ \\
Nasses simples souples & 12357,83 & 18 & $1,46 \% \circ$ \\
Nasses simples rigides & 4927,91 & 10 & $2,03 \% \circ$ \\
Nasses souples à ailettes & 973,42 & 5 & $5,14 \% \circ$ \\
\hline Total & $23461,41^{*}$ & 52 & $2,21 \% \circ$ \\
\hline
\end{tabular}

TABLEAU 6. - Bilan de l'efficacité par grand type de milieu.

\begin{tabular}{lccc}
\hline & $\begin{array}{c}\text { Nb heures- } \\
\text { pièges }\end{array}$ & Nb captures & $\begin{array}{c}\text { Taux horaire de } \\
\text { captures }\end{array}$ \\
\hline $\begin{array}{c}\text { Milieu « cours } \\
\text { d'eau» }\end{array}$ & 25409,08 & 53 & $2,09 \%$ \\
Milieu lacustre & 556,33 & 4 & $7,19 \%$ \\
\hline
\end{tabular}

objectifs différents poursuivis par nos homologues. Avec le but de suivre à moyen terme une population donnée de desmans, Melero et al. (2012) puis Esnaola et al. (2018) n'ont capturé que sur des linéaires réduits sur lesquels l'espèce était bien connue. Ils affichent des taux de capture respectifs de $10 \%$ et $25 \%$. Dans notre cas, sur la majorité des secteurs piégés (à l'exception d'Aston, de l'Aude et du Vicdessos) l'objectif était différent puisqu'il s'agissait de tester la méthode, notamment sur des sites où l'espèce n'est plus détectée et où sa présence est mise en doute. Il paraît donc logique que la répétition des captures sur ce type de site fasse baisser le taux de capture global puisque les opérations ont été négatives sur la plupart d'entre eux. À l'inverse, sur les sites considérés comme des "hot-spots» à Desman, les taux de capture se rapprochent davantage des résultats de Melero avec des chiffres supérieurs à $6 \%$. C'est notamment le cas pour la vallée d'Ossau (FR7200793) ou le secteur du Quérigut (FR7300831). Enfin, par rapport aux écarts avec les chiffres plus anciens avancés en France, la forte régression constatée de l'espèce est probablement à mettre en cause (Charbonnel et al. 2016).

Outre cette analyse, la compilation du jeu de données avait un double objectif, essayer d'apporter des éléments de protocole pour optimiser les chances de capture du Desman des Pyrénées et proposer des éléments sur la plus-value de cette méthode par rapport à la recherche de crottes comme cela avait été fait en Espagne (González-Esteban et al. 2003).

Les résultats obtenus sur les 80 opérations du jeu de données ne permettent pas de mettre en évidence de corrélations significatives entre le nombre de captures et les éléments du protocole que sont le linéaire de piégeage, le nombre de pièges posés et la durée du piégeage. Cette absence de significativité statistique peut au moins en partie s'expliquer par la constitution du jeu de données avec notamment une majorité de valeur 0 pour le nombre de captures. Il pourrait toutefois être intéressant d'étudier l'efficacité des captures en intégrant de nouvelles variables telles que la largeur et/ou la surface de cours d'eau piégé, le pourcentage de cours d'eau piégé sur une
TABLEAU 7. - Bilan de la complémentarité capture/recherche de fèces.

\begin{tabular}{cccc}
\cline { 2 - 4 } & $\begin{array}{c}\text { Recherche } \\
\text { de fèces } \\
\text { positive }\end{array}$ & $\begin{array}{c}\text { Recherche } \\
\text { de fèces } \\
\text { négative }\end{array}$ & Totaux \\
\hline $\begin{array}{c}\text { Opérations } \\
\text { avec capture }\end{array}$ & 8 & 2 & 10 \\
$\begin{array}{c}\text { Opérations } \\
\text { sans capture }\end{array}$ & 4 & 21 & 25 \\
\hline Totaux & 12 & 23 & 35 \\
\hline
\end{tabular}

section donnée ou bien encore des variables plus spécifiques au lieu de pose d'un piège (profondeur, vitesse du courant, etc.). Ces variables, notamment la largeur et la surface, pourraient aussi expliquer au moins en partie la différence avec les résultats des chercheurs espagnols dont les sites de capture sont généralement des cours d'eau de petite taille. À l'heure actuelle, les données récoltées sur le terrain ne permettent pas d'intégrer toutes ces variables mais il s'agit d'une évolution à étudier lors de prochaines opérations de captures.

Néanmoins, quelques éléments de protocole peuvent être apportés, d'abord en ce qui concerne la durée du piégeage. Si elle n'est pas directement corrélée au nombre de captures, l'analyse plus détaillée de l'horaire des 57 captures montre bien leur répartition sur la nuit entière. Plus important encore, dans certains cas où un seul Desman est capturé, cette capture unique peut intervenir très tard dans la nuit, voire à l'aube. Il apparaît donc essentiel de maintenir l'effort de piégeage sur la nuit entière surtout lorsqu'aucun desman n'est capturé sur les premières heures. De plus, cette analyse de l'horaire montre deux pics de captures globalement compris entre $22 \mathrm{~h}$ et $23 \mathrm{~h} 30$ puis entre $03 \mathrm{~h} 30$ et $06 \mathrm{~h} 00$. Il est intéressant de relever que ces deux pics correspondent aux principaux pics d'activité nocturnes de plusieurs desmans suivis par radiopistage (Fig. 6). Ces suivis tendent à montrer que tous les desmans suivis en radiopistage présentent généralement un rythme d'activité nocturne composé de deux principales phases d'activité (Lim \& Jacob 2019). Les données de captures semblent montrer que ce constat fait sur quelques individus pourrait être plus largement extrapolé.

Pour ce qui est ensuite du linéaire de piégeage, et en l'absence d'une corrélation simple entre ces deux variables, il apparaît malgré tout que le nombre de capture est plus élevé pour la classe de linéaire supérieur à $500 \mathrm{~m}$. Ce constat apporte 

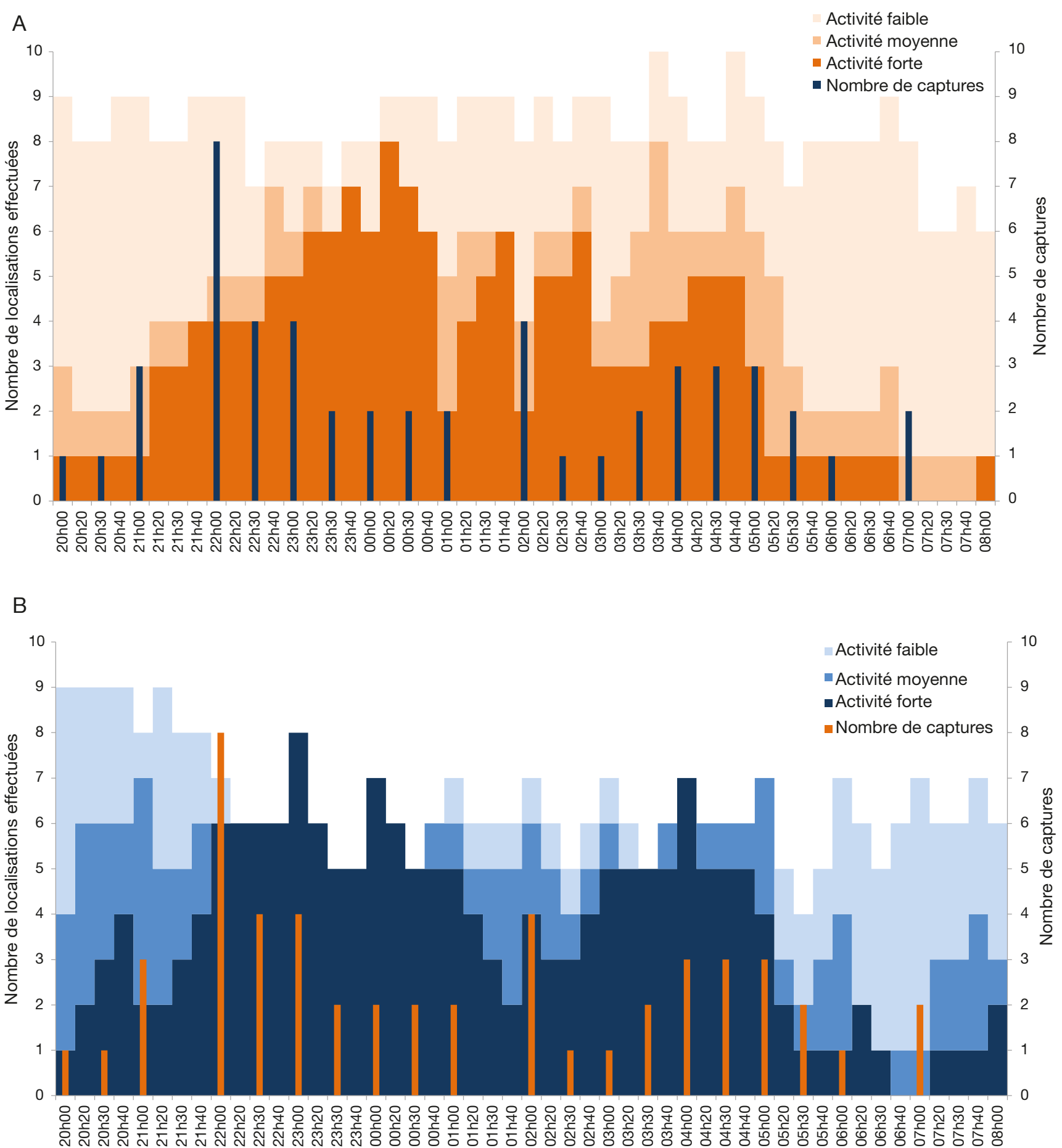

FIG. 6. - Comparaison des horaires de captures avec le rythme d'activité nocturne de deux desmans suivis par radiopistage.

donc un élément de réponse à une des questions initialement soulevées: faut-il concentrer les pièges sur un seul domaine vital théorique ou les étaler sur une surface plus importante? Les résultats obtenus semblent donc montrer que la seconde option est à privilégier pour optimiser le nombre de captures. Le Desman est désormais connu pour sa capacité à parcourir de grandes distances, y compris sur un pas de temps très resserré (Lacaze \& Blanc 2019). Aussi, le fait de couvrir un plus grand linéaire à un instant t optimise les chances de détecter l'espèce.
Par rapport au type de pièges utilisés, les nasses souples à ailettes présentent le meilleur taux de capture. Cela peut s'expliquer par le fait qu'elles sont généralement installées sur des cours d'eau de largeur petite ou moyenne, qu'elles barrent intégralement. Elles sont donc à privilégier. Néanmoins, c'est aussi un modèle qui présente plusieurs contraintes logistiques et qui ne convient qu'à des cours d'eau de largeur inférieure à $10 \mathrm{~m}$. Si, à l'image de la nasse souple simple dont elle est inspirée, la nasse à ailettes est pliable (le volume pour le transport 
n'est donc pas un facteur limitant), elle présente un poids plus élevé, d'autant plus à la fin de l'opération lorsque les ailettes sont imbibées d'eau. C'est une contrainte non négligeable pour des sites de captures non accessibles en voiture. Par ailleurs, elles sont plus longues et plus complexes à installer. Leur mise en place nécessite deux voire trois agents pour bien caler la nasse et assurer son imperméabilité avec le fond du lit du cours d'eau. Enfin, la présence des ailettes constitue un barrage pour la matière organique portée par le courant. L'accumulation qui en résulte peut déstabiliser le dispositif en pleine nuit, notamment à l'automne en période de chute des feuilles, nécessitant alors une surveillance accrue et des manipulations supplémentaires. Les nasses simples rigides, qui présentent le second taux de capture parmi les pièges utilisés aujourd'hui, ne présentent pas toutes ces contraintes mais ne sont pas pliables. Le nombre de pièges transportables est donc limité. Ainsi, le modèle de nasse semblant présenter le meilleur rapport efficacité/ contraintes sont les nasses simples souples qui sont pliables, légères et faciles à installer.

L'étude de la complémentarité de cette méthode avec la recherche de feces ne montre pas de plus-value globale des captures contrairement aux résultats de González-Esteban et al. (2003). En effet, sur la plupart des opérations, le résultat de ces deux techniques est similaire et la recherche de fèces présente davantage de résultats positifs que les captures. Les deux seules configurations où la capture a permis de corriger une prospection fèces négative se trouvaient sur des milieux lacustres. Sur ces types de milieux, où la recherche de fèces peut être délicate à cause de l'absence de zones de marquage, la capture pourrait constituer une plus-value intéressante d'autant que le taux de capture y est plus élevé. Toutefois, le jeu de données en lac demeure très faible avec seulement quatre réplicas (pour trois opérations positives!) et il n'est pas évident d'en exploiter les conclusions.

\section{CONCLUSION}

L'analyse des 80 opérations de captures menées en France depuis 2011 permet de prendre un peu de recul et d'interpréter les éléments du protocole pour le perfectionner. Trois points principaux ressortent de ce travail: maintenir la pression de capture sur l'intégralité de la nuit; privilégier des linéaires de piégeage supérieurs à 500 mètres; et favoriser dans la mesure du possible la pose de nasses à ailettes tout en sachant que les nasses souples présentent le meilleur rapport efficacité / contraintes. Pour l'établissement d'un protocole plus précis, ces informations pourraient être mises en perspective avec de nouvelles variables et avec la méthode utilisée notamment en Espagne où des études ciblées affichent des taux de captures bien supérieurs. En ce sens, les échanges d'expériences s'avéreraient très intéressants. En effet, les taux de captures faibles posent question quant à la fonctionnalité de nos pièges et des moyens qui pourraient être mis en œuvre pour l'améliorer.

Le perfectionnement de la méthode doit permettre d'optimiser les chances de captures pour mener à bien et avec de meilleurs résultats certaines études nécessitant la manipulation d'individus (CMR, radiopistage, etc.). Elle n'a pas vocation à remplacer la recherche de feces par rapport à laquelle nos résultats ne montrent pas de réelle plus-value. Là aussi, un échange avec les chercheurs espagnols serait pertinent puisque leurs résultats suggèrent l'inverse. La seule plus-value potentielle porte sur les milieux lacustres; cela constitue une piste de recherche pour continuer à optimiser la détection du Desman des Pyrénées.

\section{Remerciements}

Nous remercions les deux rapporteurs Patrick Haffner et Audrey Savouré-Soubelet pour leur relecture et leur contribution à l'amélioration de cet article. Nous remercions aussi les personnes ayant contribué à son écriture grâce à leurs relectures antérieures. Enfin, merci à toutes les personnes et partenaires ayant participé aux opérations de captures et ayant ainsi permis la constitution du jeu de données exploité ici.

\section{RÉFÉRENCES}

Aymerich P. 2018. - Distribucio de l'Almasquera a Andorra revisio del treball realitzat els anys 2002-2003. Rastres 8: 6-11.

Aymerich P., Casadesus F. \& Gosalbez J. 2001. - Distribucio de Galemys pyrenaicus (Insectivora, Talpidae) a Catalunya. Orsis 16: 93-110.

BERTRAND A. 1994. - Répartition géographique et écologie alimentaire du Desman des Pyrénées Galemys pyrenaicus (Geoffroy, 1811) dans les Pyrénées françaises. Thèse en vue de l'obtention du diplôme universitaire de recherche (Ecologie) de l'Université Paul Sabatier de Toulouse (Sciences), Laboratoire Souterain du CNRS de Moulis, $224 \mathrm{p}$.

Bertrand A. 2010. - Le Desman des Pyrénées sur le site Natura 2000 Rioumajou et Moudang - Hautes-Pyrénées. Ariège Environnement Diffusion, Boussenac, 8 p.

BERTRAND A. \& Richard P. B. 1986. - Le Desman G. pyrenaicus Distribution dans les Pyrénées françaises. $1^{\circ}$ Ariège et Haute-Garonne. Rapport méthodologique. Ministère de l'Environnement, D.P.N. \& Société de Biospéléologie, Paris, Moulis, 32 p.

Biffi M., Gillet F., LafFaille P., Colas F., Aulagnier S., Blanc F., Galan M., Tiouchichine M.-L., Némoz M., Buisson L. \& Michaux J. R. 2017. - Novel insights into the diet of the Pyrenean desman (Galemys pyrenaicus) using next-generation sequencing molecular analyses. Journal of Mammalogy 98 (5): 1497-1507. https://doi.org/10.1093/jmammal/gyx070

Charbonnel A., Blanc F., Némoz M., Buisson L., Laffaille P., Bodo A., Fournier-Chambrillon C., Fournier P., Fropier N., Lacaze V., Le Roux B., Poncet E., Pontcharraud L. \& TriBOLET L. 2017. - Outil cartographique d'alerte et cahier des charges pour la réalisation d'inventaires du Desman des Pyrénées - Outils techniques pour la prise en compte du Desman des Pyrénées dans les procédures environnementales Vol. 2. CEN MP, Toulouse, 28 p.

Charbonnel A., D’Amico F., Besnard A., Blanc F., Buisson L., Némoz M. \& LAffaille P. 2014. - Spatial replicates as an alternative to temporal replicates for occupancy modelling when surveys are based on linear features of the landscape. Journal of Applied Ecology 51 (5): 1425-1433. https://doi.org/10.1111/13652664.12301

Charbonnel A., Laffaille P., Biffi M., Blanc F., Maire A., Némoz M., SAnchez-Perez J. M., SAuvage S. \& Buisson L. 2016. - Can recent global changes explain the dramatic range contraction of an endangered semi-aquatic mammal species in 
the French pyrenees? PLOS ONE 11 (7): e0159941. https://doi. org/10.1371/journal.pone.0159941

ESNAOla A., GonZÁlEZ-ESTEBAN J., Elosegi A., ARriZABALAGA-ESCUdERO A. \& AinARTZA J. 2018. — Need for speed: preference for fast-flowing water by the endangered semi-aquatic Pyrenean desman (Galemys pyrenaicus) in two contrasting streams. Aquatic Conservation: Marine and Freshwater Ecosystems 28 (3): 600-609. https://doi.org/10.1002/aqc.2893

Fournier-Chambrillon C., Vial-Novella C., Blanc F., Durand M.-O., Lacaze V., Lafitte J., Levenard P., Le Roux B., Miquel J., Némoz M., Sourp E., Torres J. \& Fournier P. 2019. - Bilan de 10 ans d'autopsies de Desmans: causes de mortalités et autres enseignements. Colloque de restitution du LIFE+ Desman, ENSAT, Auzeville-Tolosane, Communication orale.

GonzÁlez-Esteban J., Villate I. \& Castién E. 2003. — A comparison of methodologies used in the detection of the Pyrenean desman Galemys pyrenaicus (E. Geoffroy, 1811). Mammalian Biology 68 (6): 387-390. https://doi.org/10.1078/1616-5047-00107

LACAZE V. \& BlanC F. 2019. - Structure des noyaux de populations: état des connaissances. Colloque de restitution du LIFE+ Desman, ENSAT, Auzeville-Tolosane (31-France), Communication orale.

Liм M. \& JАСов F. 2019. - Suivi du Desman des Pyrénées sur deux cours d'eau d'Ariège. Colloque de restitution du LIFE+ Desman. ENSAT, Auzeville-Tolosane (31-France), Communication orale. melero Y., Aymerich P., Luque-Larena J. J. \& Gosàlbez J.
2012. - New insights into social and space use behaviour of the endangered Pyrenean desman (Galemys pyrenaicus). European Journal of Wildlife Research 58 (1): 185-193. https://doi. org/10.1007/s10344-011-0561-7

Némoz M. \& BerTrand A. 2008. - Plan national d'Actions en faveur du Desman des Pyrénées Galemys Pyrenaicus 2009-2014. Société française pour l'étude et la protection des mammifères \& Ministère de l'Écologie, du Développement durable et de la Mer, Toulouse, $160 \mathrm{p}$.

Niethammer V. G. 1970. — Beobachtungen am Pyrenäen-Desman, Galemys pyrenaica. Bonner Zoologische Beiträge 3/4: 157-183

Peyre A. 1956. - Écologie et biogéographie du Desman (Galemys pyrenaicus G.) dans les Pyrénées françaises. Mammalia 20 (4): 405-418. https://doi.org/10.1515/mamm.1956.20.4.405

PuisséGuR C. 1935. - Recherches sur le Desman des Pyrénées. Bulletin de la Société d'Histoire naturelle de Toulouse 67: 163-227.

Queiroz A. I., Quaresma C. M., Santos C. P., Barbosa A. \& Carvalho H. 1996. - Desman distribution in Portugal, current knowledge. Council of Europe Environmental Encounters Series 25: 19-23.

RICHARD P. B. 1973. - Capture, transport and husbandry of the Pyrenean desman Galemys pyrenaicus. International Zoo Yearbook 13 (1): 174-177. https://doi.org/10.1111/j.1748-1090.1973. tb02143.x

TRutat E. 1891. - Essai sur l'histoire naturelle du Desman des Pyrénées. Édouard Privat, Toulouse, 236 p. 